\title{
YUSHKIÉVITCH SOBRE O TRATADO ALGÉBRICO DE AL-KHWARIZMI
}

\author{
Bernadete Morey \\ Universidade Federal do Rio Grande do Norte - UFRN - Brasil
}

(aceito para publicação em fevereiro de 2018)

\begin{abstract}
Resumo
O presente artigo tem como objetivo apresentar a tradução do russo para o português de um texto de A. P. Yushkiévitch, no qual ele descreve e comenta o tratado algébrico de alKwarizmi Al-Kitab al-muhtasar fi hisab al-jabr va-l-mukabala. O texto traduzido constituise em um dos tópicos tratados no livro História da Matemática na Idade Média.
\end{abstract}

Palavras-chave: Matemática, História, A. P. Yuskiévitch, Matemática islâmica medieval.

\section{[YUSHKIÉVITCH ON AL-KHWARIZMI ALGEBRIC TREATY]}

\begin{abstract}
The present article aims to present the translation from Russian into Portuguese of a text of A. P. Yushkiévitch, in which he describes and comments on the algebraic treatise of alKwarizmi al-Kitab al-muhtasar fi hisab al-jabr va-l-mukabala. The translated text is one of the topics discussed in the book History of Mathematics in the Middle Ages of the author Yushkievitch.
\end{abstract}

Keywords: Mathematics, History, A. P. Yushkiévitch, Medieval Islamic Mathematics.

\section{Introdução}

Até poucas décadas atrás, a historiografia da matemática islâmica medieval sofria por carência de bons relatos históricos. A situação tem mudando paulatinamente, sendo possível encontrar hoje um bom punhado de obras nesta temática. Por razões já explicitadas em Morey (no prelo), um dos relatos sobre a matemática islâmica medieval 
pertence à pena de Adolf Pávlovitch Yushkiévitch (1906-1933), destacado historiador da ciência e da matemática soviético, medievalista.

O presente artigo tem como objetivo disponibilizar aos leitores brasileiros um dos tópicos abordados no terceiro capítulo do livro História da Matemática na Idade Média, obra de autoria de Yushkiévitch publicada em 1961. O livro acima citado é um manual de história da matemática medieval em quatro capítulos: história da matemática na China, história da matemática na Índia, história da matemática nos países do Islã, história da matemática na Europa.

O terceiro capítulo do livro foi traduzido para o alemão e para o francês e publicado como um manual independente. Na ausência completa de um manual de história da matemática islâmica medieval no Brasil, iniciamos o trabalho de tradução desta obra do russo para o português e, apenas uma parte desta tradução está sendo o foco do presente artigo.

Além da presente introdução, o artigo tem mais três partes, sendo a primeira a apresentação de Yushkiévitch e sua obra enquanto historiador da matemática islâmica medieval. A segunda parte fala sobre as nuances do processo de tradução de uma obra para o português e a terceira parte disponibiliza ao leitor o texto em português no qual Yushkiévitch discorre sobre a álgebra de al-Khwarizmi.

\section{Yushkiévitch e a historiografia matemática islâmica medieval}

O trabalho dos historiadores da matemática soviéticos trouxe muitas contribuições originais para a área de história das ciências, e em particular, para a história da matemática islâmica medieval. Como já detalhado em (MOREY, 2017, no prelo), após a descoberta das ruínas do observatório de Ulugh Beg, vários documentos vieram foram encontrados e iniciou-se um extenso trabalho com as fontes encontradas tendo em Yushkiévitch uma das figuras centrais na organização e incentivo nos estudos e publicação das fontes encontradas, que vieram a preencher lacunas importantes na temática na historiografia islâmica medieval. São de sua autoria as publicações:

a) O tratado aritmético de Mohammed ben Musa Al-Khorezmi (Al-Khwarizmi), publicado na coletânea Trudi Instituta istorii iestestveznania e tekhniki Akademii Nauk URSS em 1954; e

b) Omar Kayyam e sua álgebra. In: Trudi Instituta istorii iestestveznania e tekhniki Akademii Nauk URSS, v. 2, Moscou, 1948

c) Sobre a quadratura da parábola de Thabit ibn Qurra. In: Istoria $i$ metodológuia iestiéstvennikh Naúk. n. 5. Moscou: MGU, 1965, p.118-125.

Em coautoria com Rozenfeld ele publicou:

d) ROZENFELD, B. A.; YUSHKIÉVITCH, A. P. Omar Kayyam. Moscou: Naúka,1965.

e) YUSHKIÉVITCH, A. P.; ROZENFELD, B. A. Djemshid Guiyacendi Al-Kashi. Chave para a Aritmética. Tratado sobre o círculo. Trad. B. A. Rozenfeld. Moscou: Gostekhisdat, 1956. 
f) ROZENFELD, B. A.; YUSHKIÉVITCH, A. P. Sobre o tratado de Nasir al-Din alTusi sobre as linhas paralelas. Istóriko-matematítcheskie Issledoványa, n. 13, 1960, p. 475-482.

g) ROZENFELD, B. A.; YUSHKIÉVITCH, A. P. Sobre o tratado de Qadi Zada alRumi sobre a determinação do seno de um grau. Istóriko-matematítcheskie Issledoványa, N. 13, 1960, p. 533-538.

No entanto, Yushkiévitch produziu uma outra obra muito importante: juntamente com Arnost Kolman (1892-1979) ele publicou a História da Matemática até o Renascimento em dois volumes, escrita para ser um manual de História da Matemática. O primeiro volume, dedicado à matemática na Antiguidade, é de autoria de Kolman. O segundo volume da obra, intitulado História da Matemática na Idade Média (Ver figura 1), é de autoria de Yushkiévitch e tem quatro capítulos: 1. Matemática na China; 2. Matemática na Índia; 3. Matemática dos Países do Islã; 4. Matemática na Europa Medieval.

Figura 1. Página de rosto do segundo volume da História da Matemática até o Renascimento (Yushkiévitch, 1961)

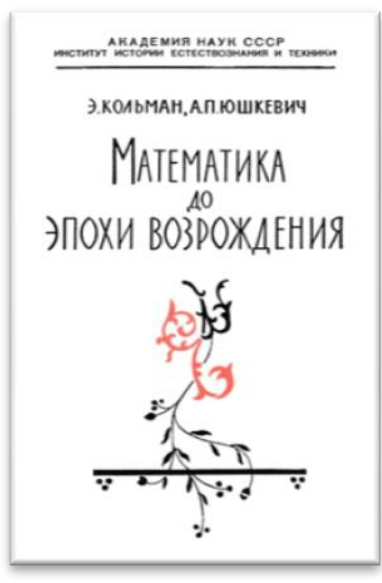

Fonte: Acervo da autora

No terceiro capítulo, em 146 páginas de texto denso, o autor incorpora os resultados de pesquisas recentes referente à história da matemática no Islã, que não apenas expõe novos fatos, mas, também leva a uma nova concepção da história da matemática no Oriente Médio e Próximo Medieval. Como já apontado em Morey (2017, no prelo), Yushkiévitch adotou uma abordagem temática ao assunto que o permitiu discorrer sobre unidades conceituais completas. Este terceiro capítulo foi traduzido para o alemão e publicado como livro independente Leipzig em 1964. A segunda edição alemã serviu de base para a tradução francesa publicada em Paris em 1976. Não encontrei, até agora, evidência da existência de tradução para o inglês. 
No que se refere ao conteúdo, inicialmente faz um breve apanhado histórico e logo a seguir se debruça em cada um dos tópicos dedicando uma seção a cada um deles que ficam assim intitulados:

1. Panorama geral;

2. Divulgação do sistema de numeração posicional decimal;

3. Frações;

4. O tratado algébrico de al-Khwarizmi;

5. A regra de três;

6. Regras da falsa posição;

7. Geometria nos trabalhos de al-Khwarizmi;

8. Os tratados de álgebra de Abu Kamil e de al-Karadji;

9. Tópicos em teoria dos números;

10. Desenvolvimento do sistema posicional;

11. Frações decimais;

12. Extração de raízes e binômio de Newton;

13. Números irracionais e teoria das congruências;

14. Problemas geométricos e equações cúbicas;

15. A teoria geométrica das equações cúbicas de Omar Khayyam;

16. O simbolismo algébrico de al-Kalaçadi;

17. Tópicos de geometria. Abu-l-Waffa

18. Estudos sobre as paralelas;

19. Secções cúbicas;

20. As novas cubaturas de ibn al-Khayçan;

21. O desenvolvimento da trigonometria;

22. O tratado dos quadriláteros de Nacir eddin at-Tusi;

23. Tabelas trigonométricas;

24. Medição do círculo de Guiacedin al-Kashi;

25. Solução algébrica da equação para a trissecção do ângulo;

26. Influência da matemática dos países islâmicos na ciência da Europa Ocidental.

O tópico de número quatro acima listado, O tratado algébrico de al-Khwarizmi, é o que foi escolhido por nós como foco do presente artigo. Falaremos mais sobre isto na sessão a seguir.

\section{Sobre o processo de tradução}

Ressaltemos inicialmente que texto escolhido para tradução, quarto tópico da listagem acima, é o comentário de Yushkiévitch sobre o tratado algébrico de al-Khwarizmi, e não o tratado propriamente dito. $\mathrm{O}$ texto foi recolhido por vários motivos:

a) sua relevância, pois, julgamos que uma grande quantidade de leitores brasileiros tais como professores de matemática em exercício ou em formação, assim como os pesquisadores iniciantes sem história da matemática não tiveram ainda oportunidade de ler um texto sobre o tratado algébrico de al-Khwarizmi; 
b) a conveniência, pois, tendo nós já assumido o compromisso de traduzir o terceiro capítulo do livro de Yushkiévitch acima mencionado, nos pareceu oportunidade apresentar um dos tópicos que já foram traduzidos;

c) por fim, as caraterísticas oportunas do texto: nem curto demais nem longo demais, sem muitos esboços, sem muitas fórmulas, o que cabe bem para o início de um trabalho de tradução.

Falemos um pouco sobre as dificuldades e das escolhas da tradução. No processo foram envolvidas três línguas: árabe, russo e português, cada uma com suas particularidades, cada uma com seu alfabeto. Isto nos levou a fazer escolhas, a começar pelo nome dos autores: o autor do texto árabe, isto é, do tratado algébrico e do autor do texto russo, isto é, do comentário sobre o tratado.

Começando pelo autor do tratado, encontramos seu nome grafado de modos distintos: al-Khwarizmi (inglês); аль-Хорезми (em russo); al-Juarismi (em espanhol). Optamos por adotar a grafia mais conhecida. Em vez de aportuguesar o nome e escrever Alquarismo ou Al-Quarismo, adotamos a bastante difundida grafia inglesa: al-Khwarizmi. Optamos também por seguir os autores que defendem a ideia que a partícula $a l$ em árabe é um artigo definido e não um nome, o que justifica ser escrito com letra minúscula. Outra opção nossa foi desprezar os sinais gráficos que acompanham o nome dos autores árabes, como, por exemplo, al-Khwārizmī, ou al-Karajī, ou Abū al-Wafă' al-Būzjanī. Mesmo ententendo que tais sinais gráficos tem uma função, achamos que carece de praticidade, uma vez que nem mesmo um teclado adequado em nossos computadores.

O autor do comentário, Yushkiévitch, Юшкевич, grafamos seu nome em português conservando os fonemas da pronúncia russa o máximo possível. Desse modo,

$\mathrm{H}=\mathrm{Yu}$;

$\amalg=\operatorname{sh}$ (poderia ser ch, em português);

ке $=$ kié;

ви $=\mathrm{vi}$;

$\mathrm{\varphi}=\mathrm{tch}$.

Portanto, Юшкевич $=$ Yushkiévitch. As traduções para outras línguas costumam obedecer ao mesmo princípio. É comum encontrarmos a grafia Youschkevitch. Isto se deve ao fato do nome do historiador russo ter se difundido na literatura ocidental a partir de suas obras em francês, língua na qual o som português de $u$ é dado pelo ditongo ou. Dito isto, podemos passar para o texto traduzido. 
соответственно $\frac{1}{6}, \frac{1}{24}$ и $\frac{1}{96}$ основной единицы (динара, дирхема II др.), для записей применялись цифры «сияка», возникшие из скорописного начертания арабских числительных. Более мелкими элементарными дробями служили данги ашаиров, тасуджи ашаиров и ашаиры ашаиров, т. е. дроби $\frac{1}{6} \cdot \frac{1}{96}, \frac{1}{24} \frac{1}{96}$, $\frac{1}{96} \frac{1}{96}$ и

Ал-Каши учит, шрежде всего, переводу обыкновенных дробей в данги, тасуджи, ашаиры и обратно. В первом случае выкладки аналогичны только что приведенным, с тем отличием, что вместо множителя 60 выступают по порядку 6, 4 п 6. Например,

$$
\frac{5}{7}=\frac{30: 7}{6}=\frac{4}{6}+\frac{8 \cdot 7}{24}=\frac{4}{6}+\frac{1}{24}+\frac{4: 7}{96}=4 \text { д. } 1 \text { т. } \frac{4}{7} \text { аш. }
$$

Для умножения и деления дробей, представленных в этой системе, пользовались таблицами, содержащими произведения кратных отдельных әлементарных дробей друг на друга. Так, чтобы умножить 5 д. 3 т. 3 аш. на 4 д. 1 т. 2 аш., находили прямо по таблице все девять промежуточных произведений, подписывали их поразрядно и, складывая, получали результат: 4 д. 1 т. 1 аш. 1 д. аш. 2 т. аш. 2 аш. аш. Деление? производили соответственно.

Алгебраический трактат ал-Хорезми. Алгебра ал-Хорезми дошла до нас в гораздо более сохранном виде, чем арифметика. В библиотеке Оксфордского университета имеется арабская рукопись алгебры, законченная в 1342 г. [104]. Кроме того, существует несколько латинских рукописей, восходящих к переводу англичанина Роберта из Честера, сделанному в Сеговии в 1145 г., и переводу итальянца Герардо из Кремоны (11141187), выполненному в Толедо $[105,106]$. Арабский текст носит заглавие «Краткая книга об исчислении алгебры и алмукабалы» («Ал-китаб ал-мухтасар фи хисаб ал-джабр ва-л-мукабала») и состоит из: 1) собственно алгебраического отдела, за которым следует маленькая глава о коммерческих сделках, - именно о простом тройном правиле по индийскому образцу, 2) небольпой геометрической главы об измерениях с некоторыми приложениями алгебры и 3 ) обширной книги о завещаниях. В латипских переводах второй и третий отделы отсутствуют. Во всех текстах есть незначительные отличия. Никаких символов ал-Хорезми не употребляет, его изложение чисто словесное и весьма пространное.

Мы упоминали уже, что основной целью ал-Хорезми при составлении трактата по алгебре было написание руководства 


\section{O tratado algébrico de al-Khwarizmi}

A álgebra al-Khwarizmi chegou até nós de forma muito mais conservada do que a aritmética. Na biblioteca da Universidade de Oxford, há um manuscrito árabe de álgebra, completado em 1342 [1]. Além disso, existem vários manuscritos latinos que remontam à tradução do inglês Robert de Chester, feito em Segovia em 1145, e à tradução do italiano Gerardo de Cremona (1114-1187), feita em Toledo [2,3]. O texto em árabe é intitulado "Um breve livro sobre o Cálculo da Álgebra e Almukabala" (Al-Kitab al-muhtasar fi hisab al-jabr va-l-mukabala) e é composto por:

a) Uma parte algébrica propriamente dita seguida de um pequeno capítulo sobre transações comerciais (na verdade, trata-se simplesmente da regra de três de acordo com o padrão indiano);

b) Um pequeno capítulo geométrico sobre medidas com algumas aplicações de álgebra e;

c) Um extenso capítulo sobre heranças.

Nas traduções latinas, a segunda e terceira partes estão ausentes. Em todos os textos existem diferenças insignificantes. Al-Khwarizmi não usa símbolos, sua apresentação é puramente verbal e ele é muito prolixo. 
к решению общежитейских задач. Этим объясняется большое место, отведенное им, в частности, задачам о завещаниях и наследствах, занимающим несколько более половины книги. Мусульманское наследственное право было (да кое-где и ныне) подчинено строгому и сложному регламенту, устанавливающему возможные доли наследников в зависимости от степени родства (жена, муж, дочь, сын, родители и т. п.) и ограничивающему права завещателя. Поэтому перед юристами возникали довольно запутанные вопросы, которые в руководствах еще усложнялись для упражнения. Задачами о завещаниях много занимались до ал-Хорезми - еще в древнем Вавилоне и после него [107].

Алгебра ал-Хорезми - это наука о решении числовых квадратных и линейных уравнений. В арифметике, говорит он, люди имеют дело с простыми числами. В алгебре рассматриваются числа трех родов: это просто число или дирхем (дирхем - от греческого драхма - денежная единица), джизр (корень) или шай (вещь) и мал (имущество, денежная сумма и т. П., также квадрат). Мал, говорит ал-Хорезми,- это произведение джизр на самого себя, а джизр - величина, которую бывает нужно умножить на самое себя.

О происхождении алгебрапческих терминов ал-Хорезми имеются различные предположения. В отделе о завещаниях и наследствах «мал» значит имущество и служит неизвестным в линейных задачах. Видимо, позднее мал стал обозначать квадрат в отличие от корня, джизр. Слово шай естественно могло быть взято для обозначения искомой величины, искомой вещи. Джизр, наверное, есть перевод санскритского мула, корень; возможна связь между словом дирхем и санскритским рупа, тоже обозначающим монету. Во всяком случае, математический смысл терминов ясен, и мы можем называть здесь джизр и шай неизвестной или корнем, мал квадратом.

Прежде всего ал-Хорезми дает классификацию рассматриваемых им шести типов линейных и квадратных уравнений и способы их решения. Затем он поясняет на примерах, как приводятся другие уравнения к шести нормальным формам. Здесь и выступают две основные операции, стоящие в названии сочинения, - ал-джкабр и ал-мукабала.

В нормальной форме все члены уравнений должны фигурировать в качестве слагаемых, а не вычитаемых. Поэтому шесть типов (их шесть, так как случаи, в которых заведомо нет положительных решений, не учитываются) таковы:

1) квадраты равны корням $a x^{2}=b x$,

2) квадраты равны числу $a x^{2}=c$,

3) корни равны числу $a x=c$, 
Já mencionamos que o objetivo principal de al-Khwarizmi, ao escrever o tratado sobre álgebra, era escrever um manual para resolver problemas quotidianos. Isso explica o lugar previlegiado atribuído, em particular, aos problemas de testamento e heranças, que ocupam um pouco mais da metade do livro. O direito hereditário muçulmano era (e, alguns lugares, continua sendo) sujeito a regulamentos rígidos e complexos que estabelecem as possíveis ações de herdeiros, dependendo do grau de parentesco (esposa, marido, filha, filho, pais, etc.) e limitando os direitos do testamenteiro. Os juízes, portanto, se viam frequentemente confrontados com questões bastante complexas, que nos manuais se complicavam ainda mais para fins de exercício. Problemas de testamento foram muito estudados antes e depois de al-Khwarizmi; foram estudados ainda na antiga Babilônia [4].

A álgebra de al-Khwarizmi é a ciência de resolver equações lineares e quadráticas. $\mathrm{Na}$ aritmética, ele diz, as pessoas estão lidando com números simples. Na álgebra são considerados números de três tipos: os números simples ou dirham (dirhem - do dracma grego - unidade monetária); o jizr (raiz) ou shay (coisa) e; o mal (propriedade, soma em dinheiro, etc., e também, o quadrado). O mal, diz al-Khorezmi, é o produto de jizra por si mesmo, enqunto que jizra é uma grandeza que às vezes pode ser multiplicada por si mesma.

Sobre a origem dos termos algébricos de al-Khorezmi existem vários pressupostos. No capítulo de testamento e heranças mal significa propriedade e serve como incógnita em problemas lineares. Aparentemente, mais tarde mal passou designar o quadrado em contraste com a raiz, jizra. A palavra shai poderia, naturalmente, ser interpretada como a quantidade procurada, a coisa procurada. Jizra, provavelmente, é a tradução do mula sânscrito, a raiz. É possível que haja ligação entre a palavra dirham e o sânscrito rupa, denotando também uma moeda. De qualquer modo, o significado matemático dos termos é claro, e podemos chamar de jizra (raiz) ou shai (coisa) à incógnita ou raiz e de mal, o seu quadrado.

Al-Khwarizmi começa dando uma classificação de seis tipos de equações lineares e quadráticas e métodos de sua resolução. A seguir ele explica com exemplos como transformar outras equações dadas em uma das seis formas normais. Aqui surgem as duas operações básicas do título da obra, al-djabr e al-mukabala.

$\mathrm{Na}$ forma normal, todos os termos das equações devem aparecer como soma, não subtração. Portanto, os seis tipos (são exatamente seis, uma vez que os casos em que não há soluções positivas, não são levados em consideração) são os seguintes:

1) quadrados são iguais a raízes, $a x^{2}=b x$;

2) quadrados são iguais a número, $a x^{2}=c$;

3) raízes são iguais a número, $a x=c$; 
4) квадраты и корни равны числу $a x^{2}+b x=c$,

5) квадраты и числа равны корням $a x^{2}+c=b x$,

6) корни и числа равны квадратам $b x+c=a x^{2}$.

Всякое другое уравнение должно быть для решения приведено к одной из этих форм. При наличии вычитаемых членов от них освобождаются с помощью ал-дкабр, т. е. восполнения, для чего к обеим частям уравнения прибавляются члены, равные вычитаемым. Далее, все подобные члены сводятся в один с помощью ал-мукабала, т. е. противопоставления. Помимо того, старший коэффициент квадратного уравнения должен быть приведен к единице, так как правила решения уравнений 4)-6) сформулированы для әтого случая.

Например, в задаче, условие которой можно записать в виде

$$
x^{2}+(10-x)^{2}=58
$$

или

$$
2 x^{2}+100-20 x=58,
$$

ал-Хорезми последовательно делает преобразования

$$
2 x^{2}+100=58+20 x \quad \text { (ал-джабр), }
$$

делит на 2 , приводит подобные члены

$$
x^{2}+21=10 x \text { (ал-мукабала) }
$$

и тем самым получает уравнение пятого типа.

Название преобразования ал-джабр, стоявшее в заглавии трактата первым, было вскоре распространено на всю науку об уравнениях. Уяе Хайям писал о. "решениях алгебры» и об «алгебраистах». Западные арабы, через посредство которых сочинение ал-Хорезми стало известным в Европе, произносили букву «джим» как г и соответственно не ал-джабр, а ал-габр. В Европе слово алгебра, в смысле названия этой науки, появляется в XIV в.

В решении первых трех типов заслуживают внимания два обстоятельства. Во-первых, уравнение $a x^{2}=b x$ ал-Хорезми трактует как линейное, не учитывая нулевого решения, в конкретных задачах неинтересного. Так поступали до XVII в. Во-вторых, - и әто весьма примечательно, - в качестве искомой неизвестной выступает не только корень уравнения, но и его квадрат. Так, определив из уравнения $x^{2}=5 x$ корень $x=5$, ал-Хорезми добавляет, что квадрат есть 25 . И в случае линейного уравнения $\frac{1}{2} x=10$ он наряду с корнем 20 приводит значение его квадрата 400; более того, в первом примере такого рода дается сразу, что корень равен 3 , и добавляется, что его квадрат есть 9 . 
4) quadrados e raízes são iguais a número, $a x^{2}+b x=c$;

5) quadraos e números são iguais a raízes, $a x^{2}+c=b x$;

6) raizes e números são iguais a quadrados, $b x+c=a x^{2}$

Qualquer equação a ser resolvida deve ser reduzida a uma destas formas. Caso haja termos subtraídos, eles são eliminados por meio da operação al-djabr, ou seja, completamento que se faz somando a ambos os membros da equação, os termos que estão sendo subtraídos. A seguir reduzem-se todos os termos semelhantes a um único por meio da transformação al-mukabala, ou seja, contraposição. Além disso, na equação quadratica o coeficiente do termo quadrado deve ser reduzido à unidade, pois, a solução das equações 4) a 6) são enunciadas somente para tal caso.

Por exemplo, no problema cujas condições podem ser escritas sob a forma

$$
\begin{gathered}
x^{2}+(10-x)^{2}=58 \\
\text { ou } \\
2 x^{2}+100-20 x=58
\end{gathered}
$$

al-Khwarizmi efetua as transformações

$$
2 x^{2}+100=58+20 x(\text { al-djabr }),
$$

divide por 2 , reduz os termos semelhantes

e com isto, obtém uma equação do quinto tipo.

$$
x^{2}+21=10 x \text { (al-mukabala) }
$$

A denominação da transformação al-djabr que apareceu primeiro no título do tratado rapidamente se difundiu para toda a ciência das equações. Omar al-Khayyam escreveu sobre "soluções de álgebra" e sobre "algebristas". Os árabes ocidentais ${ }^{1}$ por meio dos quais o tratado de al-Khwarizmi se tornou conhecido na Europa, pronunciavam a letra "djim" como "g"² e, portanto, pronunciavam al-gabr e não al-djabr. Na Europa a palavra álgebra, no sentido de denominação desta ciência, surgiu no século XIV.

$\mathrm{Na}$ resolução dos primeiros três tipos de equação, duas particularidades merecem nossa atenção. Primeiro, a equação $a x^{2}=b x$ é tratada por al-Khwarizmi como sendo linear, desconsiderando a solução nula que em problemas concretos carece de interesse. Tal procedimento continuou até o século XVII. Em segundo lugar, - e isto é notável - não apenas $x$, mas também $x^{2}$, são consideradas incógnitas procuradas. Assim, ao determinar, a partir da equação $x^{2}=5 \mathrm{x}$, a raiz $x=5$, al-Khwarizmi acrescenta ainda que o quadrado é 25. E, no caso da equação linear $\frac{1}{2} x=10$, ao lado da raiz 20, al-Khwarizmi coloca o valor de seu quadrado, 400. Além do mais, no primeiro exemplo deste tipo é dito imediatamente que a raiz é 3 e se acrescenta que seu quadrado é 9 .

\footnotetext{
${ }^{1}$ No original, западные арабы; provavelmente Yushkiévitch está se referindo aos estudiosos islâmicos da Espanha ou do Magreb. (N. T.)

${ }^{2}$ Em português, o som g como em "gato". (N. T.)
}

RBHM, Vol. 18, nº 36, p. 171-198, 2018 
Решение полных квадратных уравнений требует особого разбора. Сперва автор сообщает словесные правила выражения их корней в радикалах, а затем дает геометрические доказательства. Доказательства проводятся на числовых примерах, но имеют вполне общий характер.

Решение уравнения

$$
x^{2}+10 x=39
$$

которое, как и другие примеры ал-Хорезми, обошло чуть ли не все арабские и европейские средневековые книги по алгебре, обосновывается с помощью двух различных построений, которые оба соответствуют дополнению до квадрата. В одном из них строится искомый квадрат $x^{2}$ и на его сторонах четыре прямо-

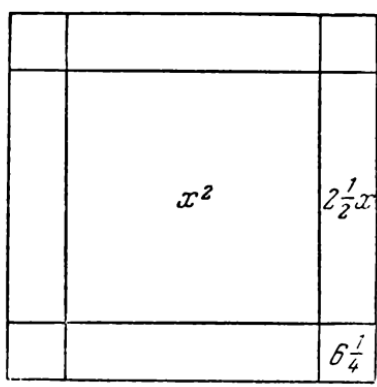

Рис. 43. угольника с высотой $\frac{10}{4}$ и в углах фигуры (рис. 43) добавляются четыре квадрата со стороной $\frac{10}{4}$. Получающийся при этом больший квадрат равен по площади $39+4\left(\frac{10}{4}\right)^{2}=$ $=64$, а сторона его, т. е. $x+2 \cdot \frac{10}{4}$, равна 8 , так что $x=3$. В случае уравнения

геометрические преобразования соответствуют алгебраическим:

$$
\begin{aligned}
x^{2}+4\left(\frac{p}{4} x\right)+4\left(\frac{p}{4}\right)^{2} & =q+4\left(\frac{p}{4}\right)^{2} \\
\left(x+2 \cdot \frac{p}{4}\right)^{2} & =q+4\left(\frac{p}{4}\right)^{2} \\
x+2 \cdot \frac{p}{4} & =\sqrt{q+4\left(\frac{p}{4}\right)^{2}}
\end{aligned}
$$

откуда и следует правило ал-Хорезми

$$
x=\sqrt{q+\left(\frac{p}{2}\right)^{2}}-\frac{p}{2}
$$

Другое геометрическое доказательство ясно из рис. 44; здесь

$$
x^{2}+2 \cdot \frac{p}{2} x+\left(\frac{p}{2}\right)^{2}=q+\left(\frac{p}{2}\right)^{2}
$$

и т. д. Обозначения на обоих рисунках - напи.

Отрицательный корень уравнения здесь и в других случаях не учитывается. 
A resolução de equações quadráticas completas exige procedimento especial. Primeiramente, o autor enuncia as regras da expressão das raízes em radicais e depois dá a demonstrações geométricas. As demonstrações apesar de serem dadas com exemplos numéricos, no entanto, são de caráter geral. A resolução da equação

$$
x^{2}+10 x=39 \text {, }
$$

que, da mesma forma de outros exemplos de al-Khwarizmi, entrou em quase todos livros medievais de álgebra árabes ou europeus, é feita com a ajuda de duas construções

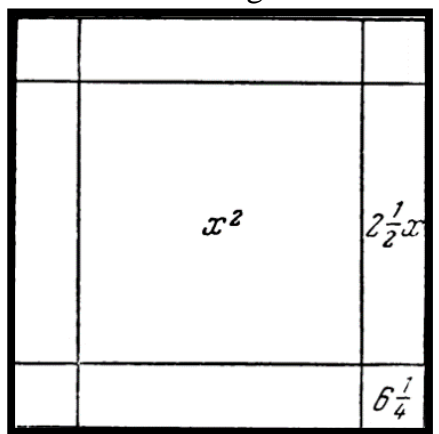

Figura 1 geométricas, ambas usando a ideia de completar o quadrado. Na primeira se constrói o quadrado procurado $x^{2}$, adjacentemente a seus lados, quatro retângulos de altura $\frac{10}{4}$ e nos cantos, quatro quadrados de lado $\frac{10}{4}$ (ver Fig.1). Obtém-se com isto um grande quadrado cuja área é $39+4\left(\frac{10}{4}\right)^{2}=64$ e cujo lado é $x+2 \cdot \frac{10}{4}$, que é igual a 8, o que nos dá $x=3$.

No caso da equação $x^{2}+p x=q$, as transformações geométricas correspondem às algébricas abaixo:

$$
\begin{gathered}
x^{2}+4\left(\frac{p}{4} x\right)+4\left(\frac{p}{4}\right)^{2}=q+4\left(\frac{p}{4}\right)^{2} \\
\left(x+2 \cdot \frac{p}{4}\right)^{2}=q+4\left(\frac{p}{4}\right)^{2} \\
x+2 \cdot \frac{p}{4}=\sqrt{q+4\left(\frac{p}{4}\right)^{2}}
\end{gathered}
$$

de onde se deduz a regra de al-Khwarizmi

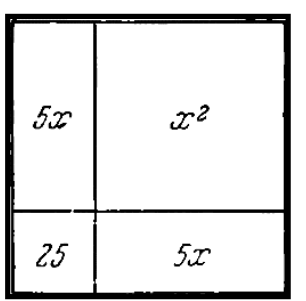

Figura 2

$$
x=\sqrt{q+\left(\frac{p}{2}\right)^{2}}-\frac{p}{2}
$$

Outro modo de fazer a demonstração geométrica (da equação $x^{2}+p x=q$, N.T) é seguindo o esquema da Figura 2 abaixo.

Teremos então $x^{2}+2 \cdot \frac{p}{2} x+\left(\frac{p}{2}\right)^{2}=q+\left(\frac{p}{2}\right)^{2}$, e assim por diante. As anotações em ambos os desenhos são nossas ${ }^{3}$. A raízes negativas aqui, assim como em outros casos, não são consideradas.

${ }^{3}$ Quer dizer, de Yushkiévitch. (N. T.)

RBHM, Vol. 18, n 36, p. 171-198, 2018 
Оставляя в стороне шестой тип, представленный уравнением

$$
x^{2}=3 x+4
$$

(в этом случае уравнение имеет один, и только один, положительный корень), рассмотрим уравнение

$$
x^{2}+q=p x .
$$

Ал-Хорезми знает, что в этом случае могут быть либо два (положительных) корня, либо один (двойной), либо ни одного (оба мнимые). Правило формулируется для уравнения

$$
x^{2}+21=10 x
$$

в следующих выражениях:

«Раздвой корни, это будет пять, и умножь это на равное себе, будет двадцать пять, и вычти из этого двадцать один, которые прибавлены к квадрату, остается четыре, извлеки из әтого корень - будет два,

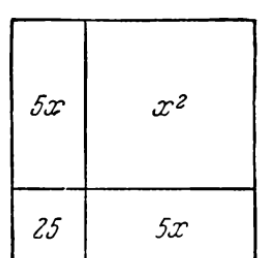

Рис. 44. и вычти это из половины корней, т. е. пяти, останется три; это и будет корень квадрата, который ты ищешь, а квадрат есть девять. А если хочешь, прибавь это к половине корней, будет семь, и әто - корень квадрата, который ты ищешь, а квадрат есть сорок девять. Если тебе встретится задача, приводящая к әтой главе, проверь ее правильность с помощью сложения, если же не так, несомненно [решение] получится с помощью вычитания. Только в әтой главе из трех глав, в которых нужно раздваивать корни, пользуются и сложением, и вычитанием. Знай также, что когда в этой главе ты раздваиваешь корни и умножаешь на равное себе, если прогзведение меньше дирхемов, прибавленных к квадрату, задача невозможна, а если оно равно дирхемам, корень квадрата равен половине корней без прибавления и отнимания» [104, стр. 11-12]. Последний случай $x=\frac{p}{2}$ специально отмечается здесь впервые в известной до сих пор литературе.

Геометрическое доказательство правила разбивается на два случая, соответственно корням

$$
x=\frac{p}{2}-\sqrt{\left(\frac{p}{2}\right)^{2}-q}
$$

$$
x=\frac{p}{2}+\sqrt{\left(\frac{p}{2}\right)^{2}-q}
$$


Deixando de lado o sexto tipo representado pela equação

$$
x^{2}=3 x+4,
$$

(neste caso a equação tem uma e apenas uma raiz positiva), nos detenhamos na equação

$$
x^{2}+q=p x .
$$

Al-Khwarizmi sabe que neste caso podem existir duas raízes (positivas), ou uma raiz (dupla) ou nenhuma raiz (ambas são imaginárias). A regra para a equação para a equação

$x^{2}+21=10 x$,

é enunciada com as seguintes palavras:

Divida as raízes ao meio, o que dará cinco e o multiplique por si mesmo obtendo vinte e cinco; do quadrado vinte e cinco subtraia vinte e um, o que dará quatro de resto; deste último extraia a raiz quadrada e obtenha dois que será subtraído da metade das raízes, ou seja, de cinco; isto dará o resto três, que será raiz do quadrado que você procura, enquanto que o quadrado será nove. E se você quiser, adicione isso à metade das raízes e ficará com sete, sendo esta a raiz do quadrado que você está procurando e o quadrado é quarenta e nove. Se você encontrar o problema que leva a este capítulo, verifique sua correção com a ajuda da adição; se não for assim, então, sem dúvida, [a solução] será obtida por subtração. Somente neste, entre os três capítulos nos quais é necessário dividir as raízes ao meio, se usa a adição e subtração. Saiba também que, quando neste capítulo, você divide as raízes ao meio e multiplica o resultado por si mesmo, se o produto for menor do que os dirhams adicionado ao quadrado, a tarefa é impossível e, se for igual aos dirhams, a raiz do quadrado é igual à metade das raízes sem adição ou subtração [1, pp. 11$12 .^{4}$

A prova geométrica da regra é dividida em dois casos, correspondentes às raízes

$$
x=\frac{p}{2}-\sqrt{\left(\frac{p}{2}\right)^{2}-q} \quad \text { e } \quad x=\frac{p}{2}+\sqrt{\left(\frac{p}{2}\right)^{2}-q}
$$

\footnotetext{
${ }^{4}$ Chamamos a atenção especial do leitor para o caso $x=\frac{p}{2}$ que aparece aqui pela primeira vez na literatura conhecida até agora. (N. do A.)
}

RBHM, Vol. 18, nº 36, p. 171-198, 2018 
Сначала подробно рассмотрен на данном числовом примере первый случай. Прямоугольник $G C D E$ со сторонами $G C=p$ и $C D=x$ (рис. 45) образован из квадрата $A B C D=x^{2}$ и приложенного к нему прямоугольника $G B A E=(p-x) x=q$. Предполагая $x<\frac{p}{2}$ (әтого ал-Хорезми не оговаривает), в $F$, середине отрезка $G C$, восставляется перпендикуляр $F H$, который продолжается на $H K=A H=\frac{p}{2}-x$. Достраиваются квадраты $G F K M=\left(\frac{p}{2}\right)^{2}$ п $J H K L=\left(\frac{p}{2}-x\right)^{2}$ По построению прямоугольники $E J L M$ и $F B A H$ с соответственно равными сторонами равны между собой. Поэтому квадрат $J H K L$, равный разности
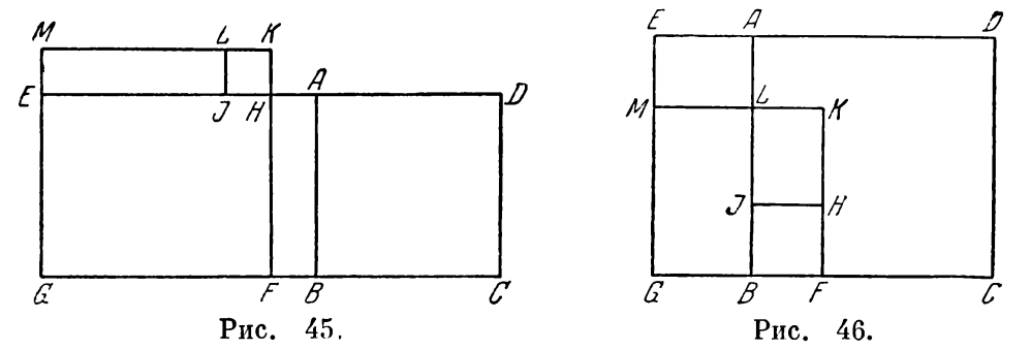

квадрата $G F K M$ и суммы прямоугольников GFHE и $E J L M$, равен данной разности величин $G F K M$ и $G B A E$, т. е.

$$
\left(\frac{p}{2}-x\right)^{2}=\left(\frac{p}{2}\right)^{2}-q
$$

Отсюда сторона $J H=A H=1^{\prime} \overline{\left(\frac{p}{2}\right)^{2}-q}$, а искомая сторона $A D=H D-H A$, т. е.

$$
x=\frac{p}{2}-\sqrt{\left(\frac{p}{2}\right)^{2}-q}
$$

Второй случай в оксфордской арабской рукописи нө разобран, сказано лишь, что больший корень получится, если прибавить к $D H$ линию $J H$. Возможно, что ал-Хорезми было известно построение и для второго случая. В некоторых латинских текстах его алгебры [106, стр. 84-87] имеются соответствующие чертежи (ср. рис. 46, где в предположении, что $x>\frac{p}{2}, F-$ середина $G C=p-$ находится внутри отрезка $B C=x$, $A B=B C$ и квадрат $B F H J$ со стороной $B F=x-\frac{p}{2}$ равен 
O primeiro caso é analisado detalhadamente no seguinte exemplo. O retângulo GCDE com lados $G C=p$, e $C D=x$ (Figura 3) gerado pelo quadrado $A B C D=x^{2}$ e pelo retângulo $G B A E=(p-x) x=q$. Supondo $x<\frac{p}{2}$ (isto não é dito por al-Khwarizmi), em F, metade do segmento GC, levanta-se a perpendicular FH que continua no segmento $H K=A H=\frac{p}{2}-x$. Finaliza-se a construção dos quadrados $G F K M=\left(\frac{p}{2}\right)^{2}$ e $J H K L=$ $\left(\frac{p}{2}-x\right)^{2}$

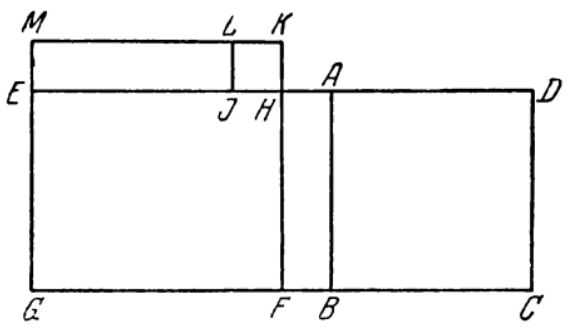

Figura 3.

Por construção os retângulos EJLM e FBAH tem lados correspondentes iguais, por isso, o quadrado JHKL é igual à diferença entre o quadrado GFKM e a soma dos retângulos GFHE e EJLM, e igual à diferença das grandezas GFKM e GBAE, ou seja,

$$
\left(\frac{p}{2}-x\right)^{2}=\left(\frac{p}{2}\right)^{2}-q
$$

Deduz-se então que o lado $J H=A H=\sqrt{\left(\frac{p}{2}\right)^{2}-q}$, e o lado procurado $A D=H D-H A$, isto é,

$$
x=\frac{p}{2}-\sqrt{\left(\frac{p}{2}\right)^{2}-q} .
$$

O segundo caso não é analisado no manuscrito de Oxford. É dito apenas que a raiz maior é obtida somando-se a linha JH a DH. É possível que al-Khwarizmi conhecesse também a construção para o segundo caso. Em alguns textos latinos de sua álgebra [3, pp. 84-87] encontram-se a figura correspondente (ver figura 4), na qual supondo-se $x>\frac{p}{2}$ e supondose que $\mathrm{F}$, o ponto médio de $\mathrm{GC}=p$, se encontra dentro do segmento $\mathrm{BC}=x, \mathrm{AB}=\mathrm{BC}$ e o quadrado BFHJ com lado $B F=x-\frac{p}{2}$ igual à diferença entre o quadrado $G F K M=\left(\frac{p}{2}\right)^{2}$ e a soma dos retângulos GBLM e JHKL. Por sua vez, GBAE $=q$, de modo que $B F=$ $\sqrt{\left(\frac{p}{2}\right)^{2}-q}$, enquanto que $x=C F+F B$; para fins de praticidade, modificamos um pouco o desenho). 
разности квадрата $G F K M=\left(\frac{p}{2}\right)^{2}$ и суммы прямоугольников $G B L M$ п $J H K L$, в свою очередь равной $G B A E=q$, так что $B F=\sqrt{\left(\frac{p}{2}\right)^{2}-q}$ и $x=C F+F B$; мы для удобства несколько видоизменили чертеж).

Изложив решение канонических типов уравнений, ал-Хорезми поясняет на примерах основные правила действий над алгебраическими выражениями: умножение одночленов и двучленов, вроде $\left(10+\frac{x}{2}\right)\left(\frac{1}{2}-5 x\right)$, приведение подобных членов в суммах и разностях, введение множителей в квадратичные радикалы или вынесение их из-под радикалов, перемножение таких радикалов. Примеры-несложные, вроде $2 \sqrt{x}=V \overline{4 x}$, $\sqrt{5} \sqrt{10}=\sqrt{50}$ и т. п. Действия сложения и вычитания ов иллюстрирует на отрезках, требуя при этом соблюдения однородности. Выражение

$$
\left(100+x^{2}-20 x\right)+\left(50+10 x-2 x^{2}\right)
$$

которое высказано, конечно, словесно, нельзя, по ал-Хорезми, представить на чертеже, так как здесь три различных рода; но его, добавляет он, легко объяснить словами.

В этих главах книги, представляющих собой как бы начала алгебраического исчисления, есть указание на существование числовых квадратичных иррациональностей, которые ал-Хорезми называет джизр асам, т. е. немой или глухой корень. Скорее всего, это перевод греческого алогос, понимаемого в смысле невыговариваемого, невыразимого словами, а не в смысле не имеющего отношения ${ }^{1}$ ). Герардо Кремонский перевел асам латинским surdus, которое сохранилось вплоть до XVIII в. наряду со словом irrationalis, встречающимся еще в древности.

Впрочем, иррациональные количества ал-Хорезми применял в очень ограниченном объеме, все примеры на уравнения у него с рациональными коэффициентами и нередко с целыми решениями. Исключениями являются лишь несколько уравнений вида $x^{2}=q$ и одно полное квадратное уравнение

$$
10 x=(10-x)^{2}, \text { т. е. } x^{2}+100=30 x,
$$

иррациональное решение которого $x=15-5 \sqrt{5}$ не приведено.

За этими главами, представляющими собой как бы начатки алгебраического исчисления в словесном изложении, следует шесть числовых задач на уравнения всех пести типов. В четырех случаях речь идет о делении числа 10 на две части по тому или

1) Напомним, что Абу-л-Вафа называл "невыговариваемые" дробп также асам (см. стр. 186). 


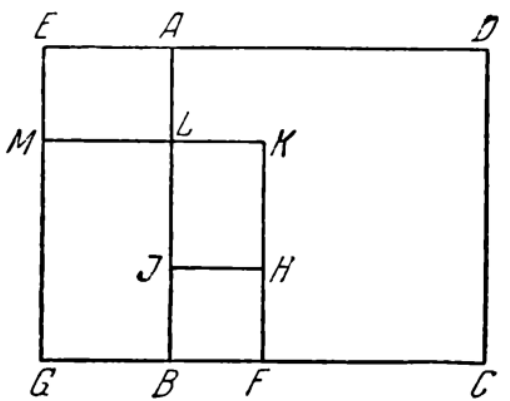

Figura 4

Expondo a solução de equações canônicas, al-Khwarizmi esclarece, por meio de exemplos, as regras das operações com expressões algébricas: a multiplicação de monônios e binômios como, por exemplo, $\left(10+\frac{x}{2}\right)\left(\frac{1}{2}-5 x\right)$, redução de termos semelhantes nas somas e subtrações, introdução de fatores em radicais quadráticos, extração de fatores dos radicais, multiplicação de radicais. Os exemplos eram simples, como

$$
2 \sqrt{x}=\sqrt{4 x} \text { ou } \sqrt{5} \sqrt{10}=\sqrt{50}
$$

e assim por diante. Al-Khwarizmi ilustra as operações de adição e subtração usando segmentos. Exige, no entanto, que seja observada a homogeneidade. A expressão

$$
\left(100+x^{2}-20 x\right)+\left(50+10 x-2 x^{2}\right),
$$

que é dada, como já sabemos, em linguagem natural, não pode ser, segundo al-Khwarizmi, representada num segmento, uma vez que ela é heterogênea (tem termos com graus distintos). No entanto, al-Khwarizmi acrescenta, é fácil explicá-la verbalmente.

Nos capítulos dedicados à introdução do cálculo algébrico, tem-se indicação da existência de irracionalidade numérica quadrada, que são chamadas por al-Khwarizmi de djizr açam, ou seja, raiz surda ou muda. Muito provavelmente, o termo é uma tradução do grego alogos compreendido mais no sentido de não pronunciável, não exprimível em palavras do que no sentido de não ter relação ${ }^{5}$. Gerardo de Cremona traduziu açam para o latim usando o termo surdus, que se conservou até o século XVIII juntamente com a palavra irrationalis encontrada ainda na antiguidade.

Aliás, al-Khwarizmi usava quantidades irracionais muito raramente; todos os seus exemplos de equações tinham coeficientes racionais e, frequentemente, tinham soluções inteiras. Como exceção a esta prática de al-Khwarizmi, pode-se apontar apenas algumas equações do tipo $x^{2}=q$ e uma equação quadrática completa $10 x=(10-x)^{2}$, ou seja, $x^{2}+100=30 x$, (cuja solução irracional $x=15-5 \sqrt{15}$ não é apontada por alKhwarizmi).

Depois destes capítulos que se constituem uma introdução ao cálculo algébrico na forma verbal (retórica), seguem seis problemas relacionados aos seis tipos de equação. Em quatro casos fala-se sobre a divisão do número $10 \mathrm{em}$ duas partes segundo uma ou outra condição, sendo os três primeiros:

\footnotetext{
${ }^{5}$ Lembremos que Abu-1-Wafa também chamou as frações "não exprimíveis" de açam (ver pág. 186). (N. do A.)
}

RBHM, Vol. 18, nº 36, p. 171-198, 2018 
иному условию, три первые таковы:

$$
\begin{aligned}
& 4 x(10-x)=x^{2}, \quad \text { T. e. } 5 x^{2}=40 x, \\
& 2 \cdot \frac{7}{9} x^{2}=10^{2}, \quad \text { т. e. } \frac{25}{9} x^{2}=100 \text {, } \\
& \frac{10-x}{x}=4, \quad \text { T. e. } \quad 5 x=10 \text {. }
\end{aligned}
$$

Заметим, что во второй задаче ал-Хорезми пользуется долями единицы и, чтобы разделить 100 на $\frac{25}{9}$, представляет $\frac{9}{25}$ как $\frac{1}{5}+\frac{4}{5} \cdot \frac{1}{5}$. В пятой задаче требуется разделить 10 на две части, сумма квадратов которых равна 58 , что приводит к уже встречавшемуся на стр. 193 уравнению

$$
x^{2}+21=10 x .
$$

В следующей главе о различных задачах решаются системы с тем же первым условием, т. е. $x+y=10$, вторые же таковы:

$$
\begin{aligned}
x y & =21, \\
x^{2}-y^{2} & =40, \\
x^{2}+y^{2}+(x-y) & =54, \\
\frac{y}{x}+\frac{x}{y} & =2 \frac{1}{6}
\end{aligned}
$$

и т. п.; только ал-Хорезми не вводит явно вторую неизвестную, а действует с частями $x$ и $10-x$, т. е. с "вещью" и "десятью без вещи». Впрочем, такие примеры не единственные. Среди многих других более интересна по условию задача, в которой требуется найти число людей $x$, если $\frac{1}{x}-\frac{1}{x+1}=\frac{1}{6}$.

Полные квадратные уравнения применяются затем в геометрическом отделе алгебры ал-Хорезми, где встречаются в промежуточной стадии решения, так как после приведения дело сводится к уравнениям первой степени. Гораздо больше в книге задач на линейные уравнения: они заполняют отдел о наследствах и завещаниях.

Мы рассмотрим одну из таких задач и увидим, что решение ал-Хорезми, несмотря на отсутствие какой-либо символики, имеет алгебраический характер, хотя, по той же причине, многословно и растянуто.

Некто, умирая, завещал четырем сыновьям по равной доле своего имущества, а одному человеку - столько, сколько составляет доля каждого из сыновей, и четверть того, что остается от трети состояния за вычетом этой доли, и один дирхем $d$. Обозначив имущество $z$, долю сына $x$ и завещанное 


$$
\begin{gathered}
4 x(10-x)=x^{2}, \text { ou seja, } 5 x^{2}=40 x, \\
2 \cdot \frac{7}{9} x^{2}=10^{2}, \text { ou seja, } \frac{25}{9} x^{2}=100, \\
\frac{10-x}{x}=4, \text { ou seja, } 5 x=10 .
\end{gathered}
$$

Notemos que no segundo problema al-Khwarizmi usa frações unitárias e, afim de dividir 100 por $\frac{25}{9}$, ele representa $\frac{9}{25}$ como $\frac{1}{5}+\frac{4}{5} \cdot \frac{1}{5}$. No quinto problema pede-se dividir 10 em duas partes cujos quadrados somados dê 58 , o que nos leva à equação $x^{2}+21=10 x$ que já encontramos anteriormente.

No capítulo seguinte, dedicado a problemas diversos, resolvem-se sistemas com a mesma primeira condição, isto é, com $x+y=10$, enquanto que a segunda condição aparece como:

$$
\begin{gathered}
x y=21 \\
x^{2}-y^{2}=40 \\
x^{2}+y^{2}+(x-y)=54 \\
\frac{y}{x}+\frac{x}{y}=2 \frac{1}{6}
\end{gathered}
$$

e assim por diante. Al-Khwarizmi não introduz explicitamente a segunda incógnita, mas ao contrário, opera com as partes $x$ e $10-x$, isto é, com a coisa e dez menos a coisa. Aliás, tais exemplos não são únicos.

Entre muitos outros, o problema mais interessante por suas condições é o problema no qual se pede encontrar o número de pessoas $x$, se $\frac{1}{x}-\frac{1}{x+1}=\frac{1}{6}$.

As equações quadráticas completas são aplicadas na seção geométrica da álgebra de al-Khwarizmi onde elas são encontradas no estágio intermediário de resolução, uma vez que, após a redução, o caso se reduz a equações do primeiro grau. No livro se encontram muito mais problemas com equações lineares: eles preenchem o capítulo sobre heranças e testamentos.

Examinemos um desses problemas e veremos que a solução al-Khwarizmi, apesar da ausência de quaisquer símbolos, tem um caráter algébrico, embora, pela mesma razão, tal solução seja prolixa e longa.

Um homem ao morrer legou aos quatro filhos partes iguais de sua propriedade e, a uma outra pessoa, tanto quanto a parcela de cada filho e mais um quarto do que sobra de um terço da propriedade após a deduzir uma parcela filial e mais um dirham d.

Indicando a propriedade como $z$, a parcela de cada filho como $x$ e a parte da pessoa que também recebeu o legado como $y$, o problema pode ser expresso pelas equações 
qеловеку $y$, мы выразили бы задачу уравнениями

$$
z=y+4 x, y=x+\frac{1}{4}\left(\frac{z}{3}-x\right)+d
$$

и, приводя, получили бы зависимость $z=5 \frac{2}{11} x+1 \frac{1}{11} d$.

То же, по существу, делает ал-Хорезми. Правило решения, говорит он, такое: надо взять $\frac{1}{3}$ имущества и вычесть из него одну долю, затем отнять $\frac{1}{4}$ того, что осталось от $\frac{1}{3}$ имущества без одной доли, и дирхем, так что останется $\frac{3}{4}$ от $\frac{1}{3}$ имущества или $\frac{1}{4}$ имущества без $\frac{3}{4}$ доли и без дирхема (иными словами, в современных обозначениях, $\frac{1}{3} z-x-\frac{1}{4}\left(\frac{z}{3}-x\right)-$ $\left.-d=\frac{1}{4} z-\frac{3}{4} x-d\right)$. Это прибавляется к $\frac{2}{3}$ имущества; тогда 11 из 12 частей имущества без $\frac{3}{4}$ доли и без дирхема равны 4 долям (иными словами, $\frac{2}{3} z+\frac{z}{4}-\frac{3}{4} x-d=\frac{11}{12} z-\frac{3}{4} x-d=$ $=4 x)$. Далее производится восполнение тремя четвертями одной доли и дирхемом, после чего 11 из 12 частей имущества равны $4 \frac{3}{4}$ долям и 1 дирхему (иными словами, $\frac{11}{12} z=4 \frac{3}{4} x+d$ ). Мы бы теперь умножили на $\frac{12}{11}$; ал-Хорезми к каждому члену прибавляет 1 из 11 его частей и получает, что имущество равно $5 \frac{2}{11}$ частям одной доли и $1 \frac{1}{11}$ дирхема.

В данной и нескольких родственных задачах дирхем играет роль параметра. В сущности, здесь мы имеем дело с серией задач на неопределенные уравнения, нередко однородные ( $d$ заранеө берется равным нулю). В ряде случаев ал-Хорезми показывает еще, как при целом $d$ получить целочисленные значения $z$ и $x$. В других задачах условие выражается уравнением с одним неизвестным, причем если оно не является имуществом в собственном смысле слова, то называется вещью.

Неизвестно, принадлежат ли ал-Хорезми самостоятельные алгебраические результаты. В начале трактата он пишет, что одни ученые имеют первенство в открытиях, другие разъясняют трудные места у своих предшественников и облегчают их 


$$
\begin{gathered}
z=y+4 x \\
y=x+\frac{1}{4}\left(\frac{z}{3}-x\right)+d
\end{gathered}
$$

e, efetuando as operações, reduzindo os termos semelhantes obtemos $z=5 \frac{2}{11} x+1 \frac{1}{11} d$. Al-Khwarizmi faz essencialmente a mesma coisa. Ele enuncia a regra para a resolução do seguinte modo:

Tem de se tomar $\frac{1}{3}$ da propriedade e dele subtrair uma parcela e depois tirar $\frac{1}{4}$ daquilo que sobrou de $\frac{1}{3}$ da propriedade menos uma parcela e um dirham, de modo que sobram $\frac{3}{4}$ de $\frac{1}{3}$ da propriedade ou $\frac{1}{4}$ da propriedade sem $\frac{3}{4}$ da parcela e sem o dirham. Em outras palavras, no simbolismo atual, $\frac{1}{3} z-x-\frac{1}{4}\left(\frac{z}{3}-x\right)-d=\frac{1}{4} z-\frac{3}{4} x-d$. Isto se adiciona aos $\frac{2}{3} d a$ propriedade. Então, 11 das 12 partes da propriedade menos $\frac{3}{4}$ da parcela e menos dirham são iguais a 4 parcelas, (em outras palavras, $\frac{2}{3} z+\frac{z}{4}-$ $\frac{3}{4} x-d=\frac{11}{12} z-\frac{3}{4} x-d=4 x$ ). A seguir adicionamos a ambos os membros três quartos de uma parcela e dirham, de modo que 11 das 12 partes da propriedade ficam iguais a $4 \frac{3}{4}$ da parcela e 1 dirham (em outras palavras $\left.\frac{11}{12} z=4 \frac{3}{4} x+d\right)$.

Atualmente, multiplicamos por $\frac{12}{11}$. Al-Khwarizmi, porém, adiciona em cada membro 1 de suas 11 partes e obtém a propriedade igual a $5 \frac{2}{11}$ partes de uma parcela e $1 \frac{1}{11}$ de um dirham.

Neste e em vários problemas relacionados o dirham desempenha o papel de parâmetro. Em essência, aqui estamos lidando com uma série de problemas em equações indefinidas, muitas vezes homogêneas ( $d$ é previamente igualado a zero). Em vários casos, al-Khwarizmi também mostra como, com $d$ inteiro, obter valores inteiros de $z$ e $x$. Em outros problemas, a condição é expressa por uma equação com uma incógnita, sendo que se não for uma propriedade no sentido próprio da palavra, então ela é chamada de coisa.

Não se sabe se al-Khwarizmi obteve ou não resultados algébricos de modo autônomo. No início do tratado, ele diz que alguns estudiosos têm primado nas descobertas, outros explicam as passagens difíceis de seus predecessores e facilitam sua compreensão, enquanto outros colocam em ordem o conhecimento já disponível corrigindo imprecisões e melhorando as ideias de seus colegas, sem acrescentar a eles e sem orgulho na alma. AlKhwarizmi pessoalmente não atribuiu a si mesmo novas descobertas. 
понимание, третьи приводят в порядок уже наличные знания, исправляя неточности и совершенствуя идеи своих товарищей, «без прибавления к ним и без гордости в душе». Себе лично ал-Хорезми новые открытия не приписывал.

Нерешенной до сих пор является проблема об источниках алгебры ал-Хорезми. В арифметике он явно следовал в основном индийским образцам (даже шестидесятеричный счет, как мы видели, он приписывал индийцам), но алгебра его представляет ряд особенностей. В индийской алгебре не встречаются геометрические обоснования правил решения квадратных уравнений или действий над алгебраическими величинами, занимающие видное место у ал-Хорезми. В отличие от индийских математиков багдадский ученый не применяет отрицательных чисел и символики. Кроме того, правило решения полного квадратного уравнения индийцы формулировали сразу для произвольного коэффициента при старшем члене и уже Брахмагупта не различал типов 4)-6). С греческой алгеброй ал-Хорезми как будто сближает геометрическое построение корней квадратных уравнений [33, III, стр. 72 и след.], но в целом его трактовка существенно отличается от геометрической алгебры «Начал» Евклида. Подлинное сходство можно увидеть только межсду вторым построением ал-Хорезми для уравнения четвертого типа и предложепием 2 книги II «Начал», геометрически представляющим формулу $(a+b)^{2}=a^{2}+2 a b+b^{2}$. Но уже первое построение ал-Хорезми того же уравнения не имеет известного нам прототипа в греческой математике. Чертеж ал-Хорезми для первого случая уравнения пятого типа напоминает построение предложения 5 книги II, но в самих выводах имеются значительные различия. Помимо того, предложение 5 не дает второго корня уравнения "с помощью сложения", как и соответственное предложение 28 книги VI «Начал». Построение ал-Хорезми для шестого типа уравнений вообще не имеет аналога у Евклида. Наконец, совершенно иной весь стиль рассуждепий и изложения у обоих авторов. Если античная геометрическая алгебра оказала влияние на ал-Хорезми, то в сильно преобразованной п приспособленной к нуждам числовой алгебры форме, исторически нигде пока не засвидетельствованной. Общим с Диофантом у ал-Хорезми является приведение квадратного уравнения к трем каноническим формам (с тем отличием, однако, что Диофант не приводит коэффицциент при квадрате неизвестного к единице). Во многом другом они расходятся, например, задачи, приводящиеся к системам

$$
x+y=a, x y=b
$$

или

$$
x+y=a, \quad x^{2} \pm y^{2}=b,
$$


Sobre quais teriam sido as fontes da álgebra de al-Khwarizmi é uma questão que permanece aberta. $\mathrm{Na}$ aritmética, ele obviamente seguiu principalmente o modelo indiano (até mesmo o cálculo sexagesimal, como vimos, ele atribuiu aos indianos), porém, sua álgebra uma série de particularidades. Na álgebra indiana, não há justificativas geométricas para as regras de resolução de equações ou operações com grandezas algébricas que ocupam um lugar proeminente em al-Khwarizmi. Ao contrário dos matemáticos indianos, o estudioso de Bagdá não usa números negativos nem símbolos. Além disso, os indianos formularam a regra para resolver a equação quadrática completa imediatamente para um coeficiente arbitrário do termo principal, e Brahmagupta já não distinguia os tipos 4) -6). A construção geométrica das raízes das equações quadráticas aparentemente aproxima alKhwarizmi da álgebra grega [33, III, pág. 72 em diante], mas, no todo, sua abordagem difere essencialmente da álgebra geométrica dos Elementos de Euclides.

Uma semelhança mais próxima só pode ser vista entre a segunda construção de alKhwarizmi para a equação do quarto tipo e a Proposição 2 do Livro II de Os Elementos, representando geometricamente a fórmula $(a+b)^{2}=a^{2}+2 a b+b^{2}$. Mas já a primeira construção de al-Khwarizmi da mesma equação não tem um protótipo conhecido na matemática grega. $\mathrm{O}$ desenho de al-Khwarizmi para o primeiro caso de uma equação do quinto tipo se assemelha à construção da Proposição 5 do Livro II, mas há diferenças significativas nas próprias conclusões. Além disso, a Proposição 5 não dá a segunda raiz da equação "por adição", assim como faz correspondente proposição 28 do Livro VI de d'Os Elementos. A construção de al-Khwarizmi para o sexto tipo de equação não tem análogo de modo algum em Euclides. Finalmente, o estilo de raciocínio e apresentação é completamente distinto de ambos os autores. Se a álgebra geométrica antiga exerceu influência em al-Khwarizmi, então o fez de forma fortemente transformada e adaptada às necessidades da álgebra numérica, historicamente em nenhum lugar ainda atestada. Em comum com Diofanto em al-Khorezm é a redução da equação quadrática às três formas canônicas (com a diferença, no entanto, que Diofanto não reduz o coeficiente do quadrado da incógnita à unidade). Em muitos aspectos, eles divergem. Por exemplo, os problemas que resultam em sistemas

ou,

$$
x+y=a, \quad x y=b
$$

$$
x+y=a, x^{2} \pm y^{2}=b,
$$


у Диофанта решаются с помощью введения вспомогательной неизвестной $\frac{x-y}{2}=z$. Впрочем, прямое влияние Диофапта маповероятно, ибо, насколько известно, первые арабские переводы Диофанта были сделаны в Багдаде христианским ученым из Баалбека (Гелиополиса) в Сирии Костой ибн Лукой ал-Ба'лабакки (ум. в 912 г. в Армении) и затем Абу-л-Вафой [108, стр. 261-264; 109, 110].

Скорее всего, ал-Хорезми были хорошо известны традиции, сложившиеся на Ближнем и Среднем Востоке и включавшие переплавленные элементы как вавилонской, так и греко-римской науки. Существует предположение, что само слово алджабр восходит через посредство сирийцев и арамейцев к ассирийскому габру-джабру-махару и, далее, к термину махаругабру, слуяившему в Вавилоне для выражения равенства двух вещей [109, стр. 275].

Тройное правило. Мы упоминали, что в алгебре ал-Хорезми приводится и разъясняется простое тройное правило. Это правило было в ходу и у других математиков. Специальное сочинение посвятил тройным правилам ал-Бируни, включив в него обобщения, сделанные индийцами (см. стр. 132-133). В трактате «Об индийских рашиках» («ФИ рашикат ал-Хинд») ${ }^{1}$ ) он рассматривает прямое и обратное правило, правила пяти, семи и более величин. В Индии, говорит ал-Бируни, он встречал задачи не более чем с одиннадцатью величинами, но их может быть любое нечетное число. Сущность правил и схема выкладок подробно поясняются на практических примерах, исходные данные которых заносятся в два столбца. Особенно удачным было рассмотрение нескольких задач на пятерное правило с одинаковыми числовыми данными, но с величинами, находящимися в различных отношениях прямой или обратной пропорциональности. В приводимых задачах находится до 17 величин. Все примеры ал-Бируни - целочисленные, но тройные правила обоснованы с помощью общей теории составных отношений; при этом он ссылается на Евклида и его комментаторов. Доказательства ал-Бируни носят поэтому общий характер. Здесь мы встречаемся с уже отмеченным весьма характерным стремлением математиков стран ислама обосновывать правила, употребляемые в практической математике, с помощью древнегреческих теорий.

Правила ложных положений. Возможно, что во времена ал-Хорезми в Багдаде уже было известно и правило двух

1) Мы знакомы с ним по неопубликованному переводу Б. А. Розенфельда. 
em Diofanto são resolvidos por meio da introdução de uma de uma incógnita auxiliar $\frac{x-y}{2}=z$.

No entanto, a influência direta de Diofante é pouco provável, pois, tanto quanto se sabe, as primeiras traduções árabes de Diofanto foram feitas em Bagdá primeiro por Kosta ibn Luka al-Ba'labakki ${ }^{6}$, estudioso cristão originário de Baalbek (Heliópolis) na Síria e depois por Abu-1-Wafa [5, pp. 261-264; 6, 7].

Muito provavelmente, al-Khwarizmi conhecia bem as tradições existentes no Próximo e Oriente Médio que incluíam elementos originários da ciência babilônica e grecoromana. Há uma suposição de que a própria palavra al-jabr veio, por meio dos sírios e aramaicos, do termo assírio gabr-jabr-mahar que, por sua vez, veio do termo mahar-gabr, que serviu na Babilônia para expressar a igualdade de duas coisas [6, p. 275].

\section{Bibliografia referenciada por Yuskiévitch}

[1] The Algebra of Mohammed ben Musa, ed. And transl. by F. Rosen, London, 1831.

[2] Liber Maumeti filii Moysi alchoarismi de algebra et almuchabala...In: G. Libri, Histoire des sciences mathématiques en Italie, vol. 1, Paris, 1838 (Tradução Latina de parte da algebra de al-Khwarizmi, pp.253-297).

[3] L. Ch. Karpinski, Robert of Chester's latin translation of the Algebra of al-Khowarizmi, NY, 1915; 2a. Ed.: L. Ch. Karpinski and J. G. Winter, Contributions to the history of science, Ann. Arbor, University of Michingan, 1930.

[4] H. Wieleteiner, Die Erbteilungsaufgaben bei Muhammed ibn Musā Alchwārazmi, Zeitschrift f. mathem. u. naturwiss. Unterricht, 53, 1922.

[5] H. Hankel, Zur Geschite der Mathematik im Altertum und Mittelalter, Leipzig, 1874.

[6] S. Gandz, The sources os al-Khowārismōs algebra, Osiris, 1, 1936.

[7] S. Gandz, The origin and development of the quadratic eqution in Babylonian, Greek and Arabic algebra, Osiris, 3, 1937.

${ }^{6}$ Morreu em 912 na Armênia

RBHM, Vol. 18, nº 36, p. 171-198, 2018 


\title{
Bibliografia
}

MOREY, Bernadete. 2017. A historiografia em russo sobre a matemática islâmica.

Conferência apresentada no XII Seminário Nacional de História da Matemática. Itajubá,

MG. Anais...

YUSHKIÉVITCH, Adolf P. 1961. Istoriya Matematiki v srednie viéka. Série Matematika do epokhi vozrojdeniya. KOLMAN, E.; YUSHKIÉVITCH, A. P. Akademya Naúk SSSR, Institut Istorii Estiestvoznanya i Tekhniki. Goçudárstvennoie Izdátelstvo Físikomatematítcheskoy Literatúri. Moskvá. (Em russo)

\author{
Bernadete Morey \\ Departamento de Matemática - UFRN - campus de \\ Natal - Brasil
}

E-mail: bernadetemorey@gmail.com 